BMJ Open

Diabetes

Research

\& Care

\section{Mendelian randomization study of serum uric acid levels and diabetes risk: evidence from the Dongfeng- Tongji cohort}

To cite: Keerman M, Yang $\mathrm{F}$ $\mathrm{Hu} \mathrm{H}$, et al. Mendelian randomization study of serum uric acid levels and diabetes risk: evidence from the Dongfeng-Tongii cohort. BMJ Open Diab Res Care 2020;8:e000834. doi:10.1136/ bmjdrc-2019-000834

- Additional material is published online only. To view please visit the journal online (http://dx.doi.org/10.1136/ bmjdrc-2019-000834).

MK and FY contributed equally.

Received 16 August 2019 Revised 3 January 2020 Accepted 14 January 2020

\section{Check for updates}

C Author(s) (or their employer(s)) 2020. Re-use permitted under CC BY-NC. No commercial re-use. See rights and permissions. Published by BMJ.

For numbered affiliations see end of article.

Correspondence to Dr Meian He;

hemeian@hotmail.com

\section{ABSTRACT}

Objective Limited Mendelian randomization (MR) studies have assessed the causal relationship between serum uric acid levels and diabetes risk. Here we investigated causality between the serum uric acid concentration and diabetes risk in Chinese population.

Research design and methods The observational analysis, based on the Dongfeng-Tongji prospective cohort $(n=15195)$ we tested the association of serum uric acid levels with incident diabetes risk. In the instrumental variable analysis, we examined the association of the genetic risk score (GRS) of serum uric acid with diabetes risk in case-control design (2539 cases and 4595 controls) via MR analysis.

Results During a mean (SD) follow-up of 4.5 (0.5) years, 1156 incident diabetes cases were identified. Compared with those in the lowest quintile of serum uric acid levels, the HRs of incident diabetes were $1.19(95 \% \mathrm{Cl} 0.96$ to 1.48), 1.12 (95\% Cl 0.90 to 1.40$), 1.38$ (95\% $\mathrm{Cl} 1.12$ to 1.70), and 1.51 ( $95 \% \mathrm{Cl} 1.23$ to 1.87 ) for Q2, Q3, Q4 and $Q 5$, respectively $(P$-trend $<0.001)$. The GRS was strongly associated with serum uric acid levels $(\beta=0.17,95 \% \mathrm{Cl}$ 0.15 to $0.19 ; P=2.81 \times 10^{-67}$ ). However, no significant association was observed between the GRS and diabetes risk (OR=1.01, $95 \mathrm{Cl} 0.95$ to $1.06 ; P=0.75)$.

Conclusions Even though serum uric acid levels were significantly associated with increased incident diabetes risk, the results did not provide evidence for a causal relationship between them.

\section{INTRODUCTION}

Type 2 diabetes (T2D) is a metabolic disorder characterized by impaired insulin sensitivity and increased insulin resistance. According to recent report, the worldwide diabetes prevalence increased from $4.3 \%$ in 1980 to $9.0 \%$ in 2014 in men, and from $5.0 \%$ to $7.9 \%$ in women, the total numbers of adults with diabetes was estimated to be 422 million in 2014. ${ }^{1}$ The pathogenesis of T2D involves genetic factors, environmental factors, and their interaction. ${ }^{2}$

Hyperuricemia was considered to precede gout and also be associated with increased risk

\section{Significance of this study}

What is already known about this subject?

- Recent decades, a number of prospective observational studies reported positive association between serum uric acid levels and incident diabetes risk

- Limited Mendelian randomization studies assessed the causal relationship between serum uric acid levels and diabetes risk with null findings

What are the new findings?

- Whether serum uric acid levels are causally correlated with the development of diabetes?

How might these results change the focus of research or clinical practice?

- Although Mendelian randomization study did not provide evidence for causality, direct experimental investigation and large randomized controlled clinical trials are needed to determine whether lowering serum uric acid treatment can reduce the risk of diabetes.

of cardiovascular disease, ${ }^{3}$ insulin resistance, ${ }^{4}$ and metabolic syndrome. ${ }^{56}$ In recent decades, a number of prospective observational studies reported positive association between serum uric acid levels and incident diabetes risk, ${ }^{7-9}$ in contrast, some indicated negative association, ${ }^{10}$ or supported bystander role of serum uric acid levels in diabetes development. ${ }^{11}$ A recent meta-analysis indicated that in the general population with each $59.48 \mu \mathrm{mol} / \mathrm{L}$ uric acid concentration increase, the incident diabetes risk increased by $13 \% .{ }^{12}$ However, whether serum uric acid levels are causally correlated with the development of diabetes, is still a matter of debate. ${ }^{13}$ Meanwhile, serum uric acid levels were significantly associated with some potential risk factors of diabetes; therefore, it is difficult to identify the independent effects of serum uric acid on diabetes risk in observational studies. 
Mendelian randomization study (MR) has been applied to identify the causal relationship between risk factors and outcomes. ${ }^{14}$ The main assumption of MR is, if a genetic variant is significantly associated with the risk factor, the association of a genetic variant with disease outcome can be evaluated independent of confounding factors. So far, limited MR studies assessed the causal relationship between serum uric acid levels and diabetes risk with null findings. ${ }^{15-17}$ In addition, most of the MR studies were conducted in European ancestry. ${ }^{15-17}$ Therefore, more MR studies to investigate the causal associations of serum uric acid with diabetes risk especially in different population were warranted.

In the current study, first we examined the associations of serum uric acid levels with incident diabetes risk in a prospective cohort study, furthermore, we selected 15 uric acid-associated single nucleotide polymorphisms (SNPs) that were identified in previous large-scale genome-wide association study as instrumental variable to conduct the MR analysis exploring whether there was causal relationship between serum uric acid levels and diabetes risk.

\section{METHODS}

\section{Study populations}

The Dongfeng-Tongji (DFTJ) cohort study is an ongoing prospective cohort study with 27009 participants at baseline. Detailed baseline profiles of this study have been reported elsewhere. ${ }^{18}$ Briefly, the DFTJ cohort was launched in 2008 in the Dongfeng Motor Corporation in Shiyan city, Hubei province, China. Between 2008 and 2010 , a total of 27009 retirees were recruited and answered the questionnaires, participated in physical examination and provided fasting blood samples. Trained investigators collected information on socio-demographics, lifestyles factors, health status and medical history via questionnaires by face-to-face interviews. During April 2013 to October 2013, the first follow-up investigation was conducted and 96.2\% (25 978/27 009) of participants were successfully followed up.

For the observational analysis, individuals with selfreported stroke, ischemic heart disease, or cancer at baseline $(n=5932)$ were excluded. To explore the association between serum uric acid levels and incident diabetes risk, participants with prevalent diabetes $(\mathrm{n}=3538)$ and with missing data of serum uric acid levels $(n=1742)$ at baseline were also excluded. Participants with missing follow-up data were further excluded $(\mathrm{n}=602)$. Finally, leaving 15195 participants $(6655$ males and 8540 females) for further observational analysis. Among the whole cohort population, genotype data were available in 7532 individuals. After exclusion of individuals with missing follow-up data $(n=127)$ or with missing uric acid data $(\mathrm{n}=271)$, leaving 7134 participants (3574 males and 3560 females) for the MR analysis. Detailed study design and inclusion and exclusion criteria for the current research were summarized in figure 1

All participants provided written informed consent.

\section{Assessment of diabetes mellitus}

Diabetes were defined if one or more of the following criteria were met: (1) fasting plasma glucose $(\mathrm{FPG}) \geq 7.0 \mathrm{mmol} / \mathrm{L}$; (2) self-reported physiciandiagnosed diabetes or currently taking anti-diabetic medication; (3) hemoglobin $\mathrm{A}_{1 \mathrm{c}}\left(\mathrm{HbA}_{1 \mathrm{c}}\right) \geq 6.5 \%$. Cut-off value of FPG and $\mathrm{HbA}_{1 \mathrm{c}}$ for diagnosis of diabetes were recommended by the American Diabetes Association. ${ }^{19}$ In the present study, we did not differentiate types of diabetes.
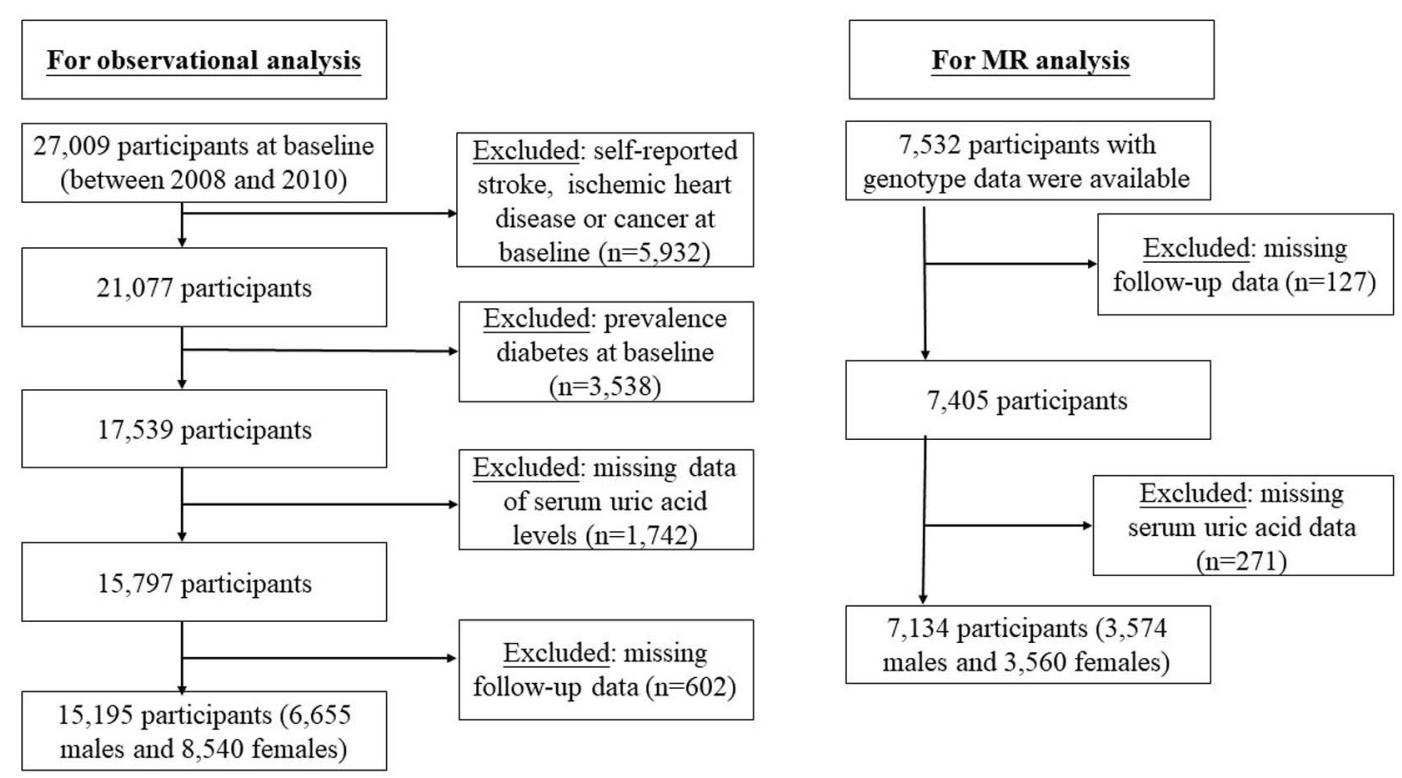

Figure 1 Study design and study population for the current research. MR, Mendelian randomization study. 


\section{Assessment of hyperuricemia}

Hyperuricemia was identified according to any of the following criteria: self-reported physician-diagnosed gout or taking anti-gout medication or males with serum uric acid levels $\geq 420 \mu \mathrm{mol} / \mathrm{L}$ or females with serum uric acid levels $\geq 360 \mu \mathrm{mol} / \mathrm{L}^{20}$ According to the quintiles of gender-specific distribution, serum uric acid levels were grouped into five categories:<260, 260-298, 299-335, 336-384 and $\geq 385 \mu \mathrm{mol} / \mathrm{L}$ for males; and <202, 202-236, 237-266, 267-308 and $\geq 309 \mu \mathrm{mol} / \mathrm{L}$ for females.

\section{Measurement of uric acid levels and other covariates}

Baseline information on socio-demographics, lifestyles factors, health status, and medical history were obtained by face-to-face interviews through questionnaires. Standing height, body weight and waist circumference (WC) were measured with light indoor clothing and without shoes. Physically active was defined as having exercise regularly for at least $20 \mathrm{~min}$ each time in the past 6 months. Current smokers were those who smoke at least one cigarette per day and continued more than half a year. Ex-smokers were those who have quitted smoking for more than half a year. Current alcohol consumers were those who drink at least one time per week and continued more than half a year. Abstainers were those who have stopped drinking for more than half a year. Prevalent hypertension was defined as blood pressure $\geq 140 / 90 \mathrm{~mm} \mathrm{Hg}$, or taking anti-hypertensive medication or self-reported of physician-diagnosed hypertension. Body mass index (BMI) was calculated by weight in kilograms divided by square of height in meters. We calculated estimated glomerular filtration rate (eGFR) using the Chronic Kidney Disease Epidemiology Collaboration equation $^{21}$. Levels of serum uric acid, creatinine and serum lipids were determined using ARCHITECT Ci8200 automatic analyzer (ABBOTT Laboratories, Abbott Park, Illinois, USA). FPG concentrations were measured with Glucose Oxidase method by Aeroset Automatic analyzer (Abbott Laboratories). $\mathrm{HbA}_{1 \mathrm{c}}$ levels were measured with high-performance liquid chromatography D-10 system (Bio-Rad Laboratories. Hercules, California, USA).

\section{Genotyping and quality control}

Reported SNPs were downloaded from website of Genome-Wide Association Studies (GWAS) catalog (https://www.ebi.ac.uk/gwas/). We searched the website of GWAS catalog for all relevant studies until March 8th, 2018 by searching for 'uric acid' or 'urate'. Finally, 72 SNPs passed the significant threshold of $<5 \times 10^{-8}$ in previous large-scale genome-wide association studies. ${ }^{22-26}$ Detailed information of individual SNPs was described in online supplementary table 1 . We selected SNPs that passed quality control criteria, including (1) minor allele frequency $(\mathrm{MAF})>0.05$; (2) Hardy-Weinberg equilibrium test $(P$ values $\geq 0.05)$; and (3) call rate $>95 \%$. In addition, after linkage disequilibrium test and conditional analysis, we chose SNPs that passed the threshold of at least $\mathrm{p}<0.05$ in the present study. Detailed results of linkage disequilibrium test were displayed in online supplementary table 2. Finally, 15 SNPs mapped in or near PDZK1 (rs1471633), TRIM46 (rs11264341), SLC17A1 (rs1165151), RFT1 (rs6770152), NRXN2 (rs478607), SLC2A9 (rs11722228 and rs3775948), ABCG2 (rs2231142 and rs3114018), LRP2 (rs2544390), STC1 (rs17786744), HNF4G (rs2941484), SLC22A12 (rs505802), IGF1R (rs6598541) and BCAS3 (rs2079742) were selected for further Mendelian randomization analysis. The genotypes of the selected 15 SNPs were derived from the Affymetrix Genome-Wide Human SNP Array 6.0 Chips and Illumina Infinium OmniZhonghua-8 Chips. The genotype procedure and quality control have been described in detail in previous studies. ${ }^{2728}$

\section{Statistical analysis}

In observational analysis, we used Cox proportional hazard regression model to estimate the multivariable adjusted HRs of serum uric acid levels and hyperuricemia on incident diabetes risk. We adopted linear regression model to estimate the association of individual SNPs and the GRS with serum uric acid levels. A conditional analysis was applied with linear regression model to examine the independence of correlated SNPs. We examined the association of the GRS with potential confounders using linear regression (for quantitative variables) and logistic regression (for qualitative variables) model. Logistic regression model was also applied to calculate OR of diabetes risk for individual SNPs and the GRS with additive genetic models. Furthermore, the expected effect sizes $\left(\beta_{\mathrm{E}}\right)$ of individual SNPs and the GRS on diabetes risk were calculated based on the effect sizes $\left(\beta_{\mathrm{GB}}\right)$ of individual SNPs and the GRS on serum uric acid levels and the observed effect sizes $\left(\beta_{\mathrm{BD}}\right)$ of serum uric acid levels on diabetes risk: $\beta_{\mathrm{E}=} \beta_{\mathrm{GB} \times} \beta_{\mathrm{BD}}{ }^{29}$ The differences between expected effect size and observed effect size were tested by students' $\mathrm{t}$ test. ${ }^{30}$ We further conducted weighted median, inverse-variance weighted, and MR-Egger methods to estimate the causal effect of serum uric acid levels on diabetes risk. ${ }^{31}$ In addition, we calculated F statistic for instrumental variable selection, F statistic greater than 10 is evidence of a strong instrumental variable. Detailed information of F statistic calculation was summarized in online supplementary table 3. Statistical analyses were performed with SAS V.9.1 and R V.3.5.0 (R Project for Statistical Computing, Package: 'Mendelian Randomization').

\section{RESULTS}

In the observational analysis, a total of 15195 individuals were included in the current study. The mean (SD) of age was 61.5 (7.6) years, and $43.8 \%$ were males. The mean (SD) of baseline serum uric acid levels were 287.18 (79.97) $\mathrm{mmol} / \mathrm{L}$ among the whole population, 304.94 (82.37) $\mu \mathrm{mol} / \mathrm{L}$ for those who developed incident diabetes and $285.71(79.59) \mu \mathrm{mol} / \mathrm{L}$ for those did not develop diabetes during the follow-up period. During a 
mean (SD) follow-up of $4.5(0.5)$ years, 1156 incident diabetes cases were identified. The incidence rate of diabetes in the present population was $7.6 \%$, and $44.8 \%$ of them were males. Detailed baseline characteristics of the participants stratified by quintiles of baseline serum uric acid levels were summarized in table 1 . Individuals with higher serum uric acid concentration tended to have higher levels of BMI, WC, blood pressure, FPG, triglyceride, total cholesterol, low density lipoproteincholesterol, serum creatinine, and higher prevalence rate of hypertension and hyperlipidemia, in contrast, lower levels of high density lipoprotein-cholesterol (HDL-C) and eGFR. Baseline characteristics of the individuals included in the analysis and those lost to follow-up were similar (online supplementary table 4). In the MR analyses, a total of 7134 participants (2539 prevalent diabetes cases and 4595 non-diabetic controls) with genotype data were included. The mean (SD) of age was $63.4(7.5)$ years and $50.1 \%$ were males. Online supplementary table 5 presented the characteristics of participants in the MR analysis.

\section{Association of serum uric acid concentration with incident diabetes risk}

In the observational analyses, compared with those in the lowest quintile of serum uric acid levels, individuals in the higher quintiles of uric acid had higher incident diabetes risk. The HRs of incident diabetes were 1.19 (95\% CI 0.96, 1.48), 1.12 (95\% CI 0.90, 1.40), 1.38 (95\% CI 1.12, 1.70), and 1.51 (95\% CI 1.23, 1.87) for individuals in Q2, Q3, Q4, and Q5, respectively ( $P$-trend $<0.001$; Model 2) (table 2). The observed association attenuated but remained significant after further adjustment for baseline FPG levels ( $P$-trend=0.003; Models 3 ) (table 2 ). When modeling as continuous variable, per SD (79.96 $\mu \mathrm{mol} / \mathrm{L}$ ) of serum uric acid levels increase was significantly associated with 1.10 fold higher incident diabetes risk after further adjustment for potential covariates and baseline FPG levels (95\% CI 1.02 to 1.17 ; p=0.007) (table 2).

\section{Association of genetic variants with serum uric acid levels and diabetes risk}

No significant association was observed between the GRS and potential confounders of diabetes except for smoking status $(P=0.02)$ with marginal significance (online supplementary table 6 ). All selected fifteen variants were significantly and positively associated with serum uric acid concentration. The effects attenuated but remained significant after adjustment for confounders, including age, sex, BMI, smoking status, drinking status, physical activity, and eGFR ( $\beta$ ranged from $0.03 \mu \mathrm{mol} / \mathrm{L}$ to 0.17 $\mu \mathrm{mol} / \mathrm{L}$ ) (table 3). The individual SNPs were not associated with diabetes risk in both univariate and multivariable models (table 3 and online supplementary table 7 ).

For the association of GRS with normal scores of uric acid levels and diabetes risk, $\beta$ and OR were both obtained by per $1 \mathrm{SD}$ increase in genetic risk score.
In the MR analysis, we calculated GRS by summing the number of uric acid-raising alleles. The results indicated that higher GRS was strongly associated with higher serum uric acid concentration $(\beta=0.17,95 \%$ CI 0.15 to $0.19 ; P=2.81 \times 10^{-67}$ ) (table 3 ). The GRS accounted for $2.9 \%$ of uric acid variance in the present population. No significant association was observed between the GRS and diabetes risk $(\mathrm{OR}=1.01,95 \% \mathrm{CI} 0.95$ to $1.06 ; P=0.75)$ (table 3). The associations of the individual SNP or GRS with diabetes risk were not different from the expected associations based on the observed associations between these SNPs (or GRS) and serum uric acid levels and the association between uric acid levels and diabetes risk (table 3). Similarly, we did not obtain evidence for causal association of GRS and diabetes risk when three different methods (weighted median based, inverse-variance weighted, and MR-Egger) were conducted (online supplementary table 8 ).

\section{DISCUSSION}

\section{Main findings}

In the current large prospective cohort study we found that higher levels of serum uric acid were independently and significantly associated with an increased risk of incident diabetes. However, our findings did not support the causal role of serum uric acid levels in diabetes.

\section{Observational analysis between serum uric acid levels and diabetes risk}

Most of the previous observational studies supported positive association between serum uric acid levels and diabetes risk. ${ }^{12}{ }^{32-35}$ Our results were in line with these findings. Moreover, in the present study the positive associations attenuated but remained significant after further adjustment for baseline fasting glucose levels, indicating the independent effects of uric acid on diabetes risk, consistent with previous study. ${ }^{9}$ Several potential mechanisms might account for the positive association between them. Insulin resistance is considered to be the main pathogenic mechanism underlying type 2 diabetes, ${ }^{36}$ and is mediated partly by inflammation and oxidative stress. ${ }^{37}$ Studies showed that serum uric acid played an important role in inducing inflammation and oxidative stress in adipocytes, ${ }^{38} 39$ which were closely correlated with the occurrence of type 2 diabetes. ${ }^{40} 41$ Moreover, experimental studies demonstrated that uric acid could directly impair insulin signaling by interfering with it at receptor level ${ }^{42}$ and raising uric acid with fructose could cause insulin resistance. ${ }^{43}$ In addition, evidence from human intervention studies indicated that uric acidlowering treatment could improve insulin resistance in chronic heart failure subjects with hyperuricemia ${ }^{44}$ and decrease diabetes risk in patients with gout, ${ }^{45}$ while other intervention study showed that urate acid-lowering agent could not improve endothelial function in type 2 diabetes cases. ${ }^{46}$ Although numerous studies indicated that uric acid plays an important role in the development 


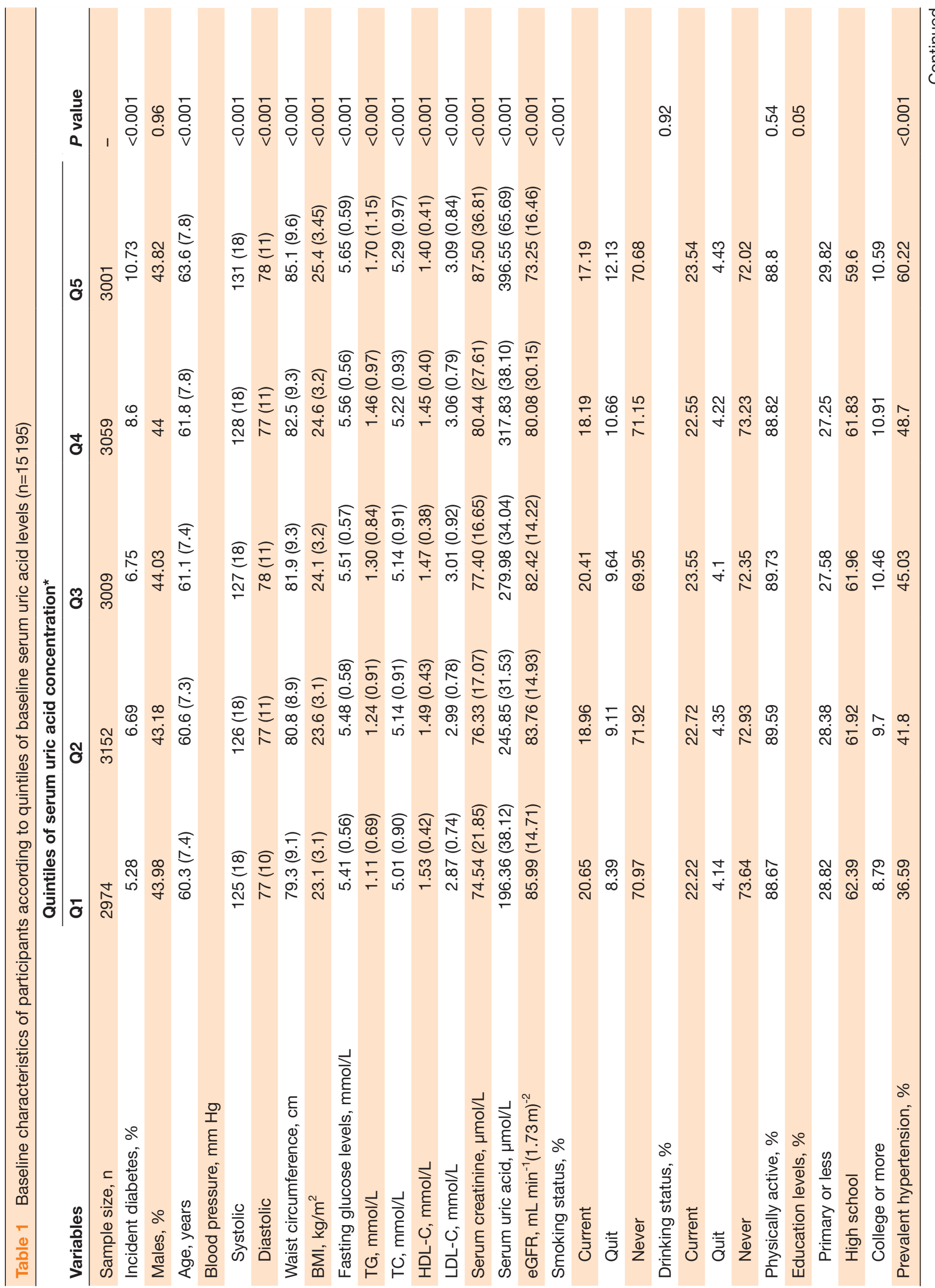


of diabetes, ${ }^{4345} 47$ however, evidences also suggested that uric acid was also related to diabetes risk markers ${ }^{5} 4849$ and uric acid might only play bystander role in diabetes risk. Therefore, whether there were causal associations between serum uric acid and diabetes risk still remained to be investigated.

Instrumental analysis between genetic variants and diabetes risk

MR analysis is a valid approach to explore causality. So far, three MR studies assessed the causal relationship between serum uric acid levels and diabetes risk with null findings. ${ }^{15-17}$ Among these MR studies, two studies conducted in Europeans found null causal associations of uric acid on diabetes risk with eight and 24 SNPs as instrumental variables respectively. ${ }^{15} 16$ Another MR study ${ }^{17}$ enrolled both Europeans and South Asians found that circulating serum urate levels were not causally correlated with cardio-metabolic diseases including T2D. However, this study was conducted using summary level data and did not control for potential mediators between genotypes and disease risk. Therefore, more MR studies to investigate the causal associations of serum uric acid with diabetes risk especially in different populations were warranted.

MR method is applied in epidemiology to test the causality of associations between risk factors and outcomes ${ }^{14}$ which is based on the random assortment of alleles/genotypes transferred from parent to offspring at the time of gamete formation. The results of MR study are based on the following assumptions. First, single genetic variants and/or instrumental variables (GRS) should be associated with risk factor of interest. In the present study, all SNPs we selected were strongly associated with serum acid concentrations in large-scale genome-wide association studies ${ }^{22-26}$ and also validated in the present population. In addition, all SNPs we selected were not in linkage disequilibrium with each other and conditional analysis indicated these SNPs were independently associated with serum uric acid levels. Second, genetic variants should be independent of potential confounders. No significant associations were observed between the GRS and potential confounders, except for smoking status with marginally significance. When we additionally adjusted for smoking status in instrumental variable estimates on diabetes risk, the null-effect remained (online supplementary table 7). Finally, single genetic variants and/or instrumental variables (GRS) should affect the outcomes only through the risk factor of interest. This assumption depends on background knowledge of the underlying biology. Most of the SNPs we selected are mapped in or near genes responsible for cellular transport of uric acid, which suggests that these SNPs might directly influence serum uric acid levels. However, one previous phenomewide association study found that missense SNP rs2231142 in $A B C G 2$ gene was also correlated with blood pressure levels and protoporphyrin levels, ${ }^{50}$ suggesting pleiotropy. Besides, the initial reports on SLC2A9 described 


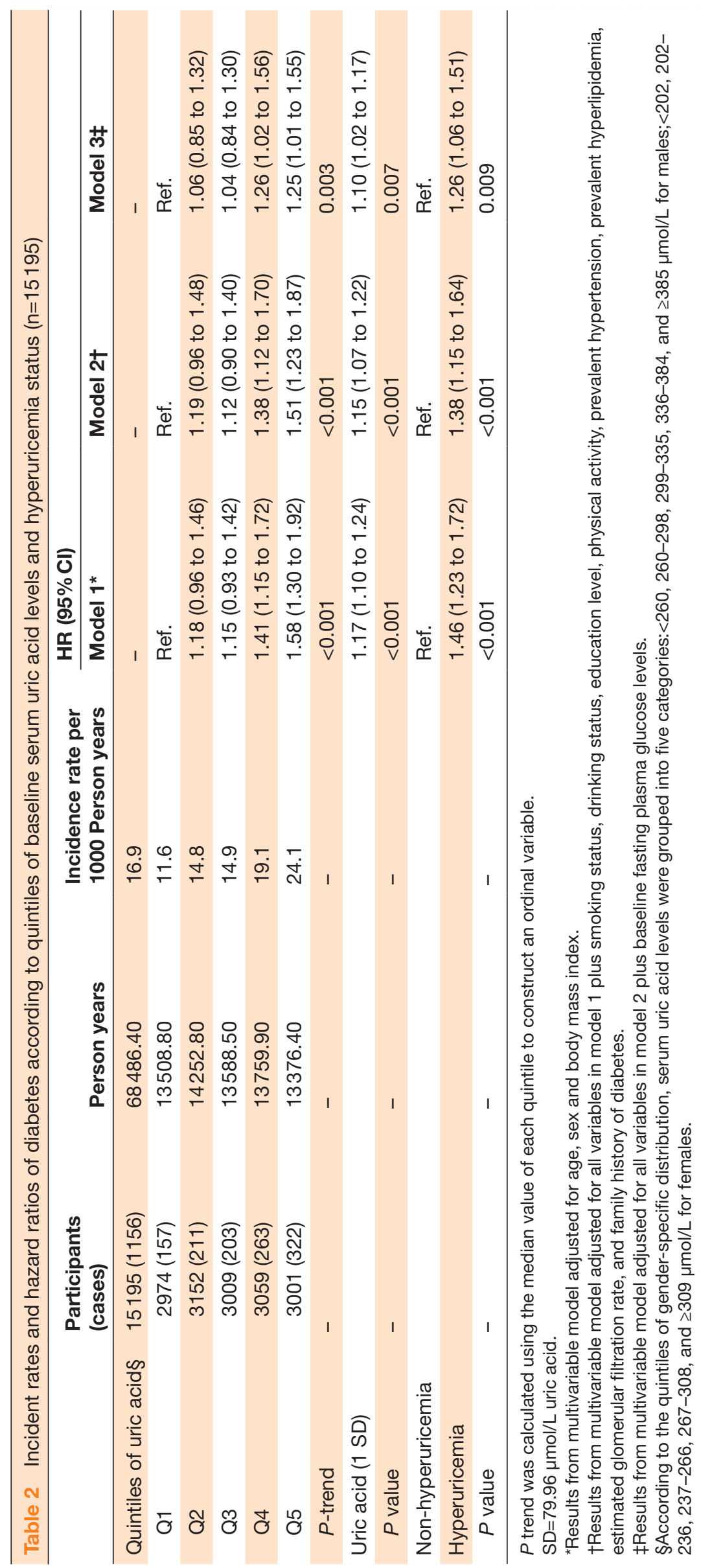




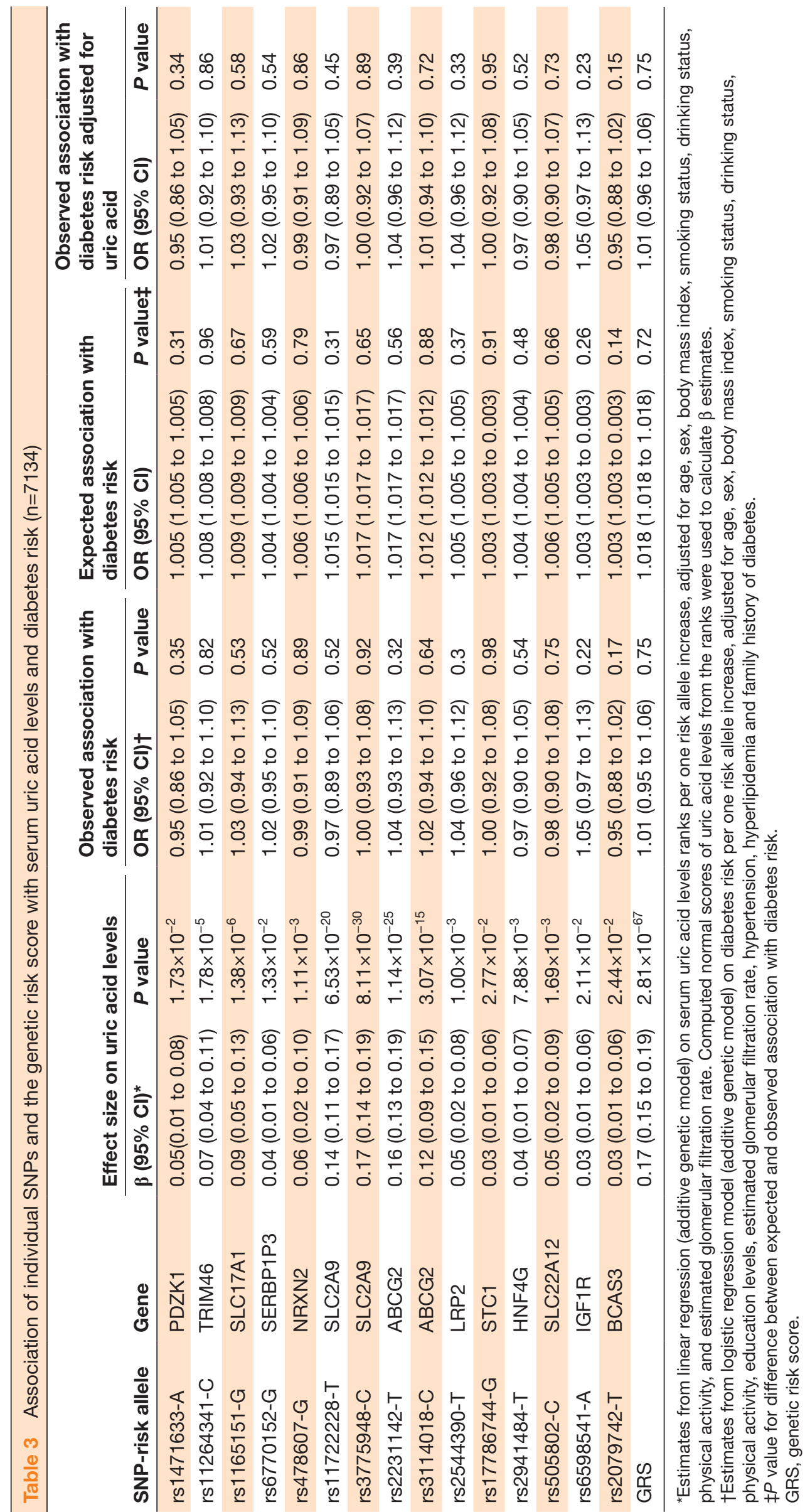


a fructose and glucose transport activity, ${ }^{51} 52$ indicating pleiotropy nature of SLC2A9 gene. However, when we additionally excluded these potential pleiotropy SNPs in $A B C G 2$ and SLC2A9 from instrumental analysis, the null-effect still remained (online supplementary table 9). Additionally, we used the MR-Egger regression methods to test the potential pleiotropy nature of SNPs. ${ }^{31}$ The intercept term estimated from MR-Egger regression was cantered at the origin with a CI including the null, suggesting that our results were not being influenced by pleiotropy. In the current study, no significant association was observed between the genetic score and diabetes risk $(\mathrm{OR}=1.01$, $95 \mathrm{CI} 0.95$ to $1.06 ; \mathrm{p}=0.75)$, which was in line with the results of previous MR studies. ${ }^{15-17}$ In addition, when we estimated the association between the genetic score and diabetes risk based on the previous reported SNPs that independently associated with serum uric acid levels in Chinese population ${ }^{24}$, the null-effect still remained (online supplementary table 10). This might be due to the relatively small sample size in MR analysis, the limited SNPs included and the relative minor effects of SNPs on uric acid levels.

\section{Strengths and limitations}

Strengths of the current study included its large sample size, prospective design, and standardized measurements of diabetes risk factors. Both in observational analysis and instrumental analysis, we took a wide spectrum of known or potential confounders of diabetes risk into consideration, including socio-demographics, lifestyles factors, and biochemical factors. In addition, we used multiple uncorrelated SNPs associated with serum uric acid levels to calculate GRS, which increased the precision of the estimate. However, there are several potential limitations tha ould be considered in the current study. First, in line with previous MR studies, ${ }^{15} 16$ in the current study the GRS only explained $2.9 \%$ of serum uric acid variation. Finding more uric acid susceptibility loci was warranted to investigate the causal associations in future studies. Second, our analyses were confined to middle-aged and elderly Chinese population, which limits generalizability to other ancestries or the young population. Finally, we cannot exclude the possibility of residual confounding, although we took possible risk factors of diabetes into consideration.

In conclusion, even though serum uric acid levels were significantly associated with increased incident diabetes risk, the results from Mendelian randomization study did not provide evidence for a causal relationship between serum uric acid levels and diabetes risk.

\section{Author affiliations}

${ }^{1}$ Department of Occupational and Environmental Health and State Key Laboratory of Environmental and Health (incubating), School of Public Health, Tongji Medical College, Huazhong University of Science and Technology, Wuhan, China ${ }^{2}$ Department of Nutrition and Food Hygiene, School of Public Health, Tongji Medical College, Huazhong University of Science and Technology, Wuhan, China ${ }^{3}$ Department of Cardiovascular Disease, Dongfeng Motor Corporation General Hospital, Shiyan, China
Acknowledgements The authors thank the staff and the participants of the Dongfeng-Tongji (DFTJ) cohort study for their contributions.

Contributors MK, FY and MH took part in the study design and data collection. $\mathrm{MH}$ supervised the study. MK, FY and MH drafted the manuscript. All authors critically revised the manuscript for important intellectual content and approved the version to be published. All authors had access to the data, commented on the report drafts and approved the final submitted version.

Funding The grants from the National Key R\&D Program of China (2017YFC0907501 and 2016YFC0900800), the Program for HUST Academic Frontier Youth Team (2017QYTD18), and the National Natural Science Foundation (grant NSFC-81522040 and 81473051).

Competing interests None declared.

\section{Patient consent for publication Not required.}

Ethics approval The Medical Ethics Committee of the School of Public Health, Tongji Medical College, Huazhong University of Science and Technology, Wuhan, China and the Ethics Committee of Dongfeng General Hospital, Shiyan, China.

Provenance and peer review Not commissioned; externally peer reviewed.

Data availability statement All data relevant to the study are included in the article or uploaded as supplementary information. Data are available upon reasonable request.

Open access This is an open access article distributed in accordance with the Creative Commons Attribution Non Commercial (CC BY-NC 4.0) license, which permits others to distribute, remix, adapt, build upon this work non-commercially, and license their derivative works on different terms, provided the original work is properly cited, appropriate credit is given, any changes made indicated, and the use is non-commercial. See: http://creativecommons.org/licenses/by-nc/4.0/.

ORCID ID

Meian He http://orcid.org/0000-0002-2096-921X

\section{REFERENCES}

1 NCD Risk Factor Collaboration (NCD-RisC). Worldwide trends in diabetes since 1980: a pooled analysis of 751 population-based studies with 4.4 million participants. Lancet 2016;387:1513-30.

2 Kahn SE, Cooper ME, Del Prato S. Pathophysiology and treatment of type 2 diabetes: perspectives on the past, present, and future. The Lancet 2014;383:1068-83.

3 Kanbay M, Segal M, Afsar B, et al. The role of uric acid in the pathogenesis of human cardiovascular disease. Heart 2013;99:759-66.

4 Johnson RJ, Nakagawa T, Sanchez-Lozada LG, et al. Sugar, uric acid, and the etiology of diabetes and obesity. Diabetes 2013;62:3307-15.

5 McKeigue PM, Campbell H, Wild S, et al. Bayesian methods for instrumental variable analysis with genetic instruments ('Mendelian randomization'): example with urate transporter SLC2A9 as an instrumental variable for effect of urate levels on metabolic syndrome. Int J Epidemiol 2010;39:907-18.

6 Dai X, Yuan J, Yao P, et al. Association between serum uric acid and the metabolic syndrome among a middle- and old-age Chinese population. Eur J Epidemiol 2013;28:669-76.

7 Dehghan A, van Hoek M, Sijbrands EJG, et al. High serum uric acid as a novel risk factor for type 2 diabetes. Diabetes Care 2008;31:361-2.

8 Bhole V, Choi JWJ, Kim SW, et al. Serum uric acid levels and the risk of type 2 diabetes: a prospective study. Am J Med 2010;123:957-61.

9 Juraschek SP, McAdams-Demarco M, Miller ER, et al. Temporal relationship between uric acid concentration and risk of diabetes in a community-based study population. Am J Epidemiol 2014;179:684-91.

10 Taniguchi $\mathrm{Y}$, Hayashi T, Tsumura K, et al. Serum uric acid and the risk for hypertension and type 2 diabetes in Japanese men: the Osaka health survey. J Hypertens 2001:19:1209-15.

11 Tsouli SG, Liberopoulos EN, Mikhailidis DP, et al. Elevated serum uric acid levels in metabolic syndrome: an active component or an innocent bystander? Metabolism 2006;55:1293-301.

12 Xu Y-L, Xu K-F, Bai J-L, et al. Elevation of serum uric acid and incidence of type 2 diabetes: A systematic review and metaanalysis. Chronic Dis Transl Med 2016;2:81-91.

13 Johnson RJ, Merriman T, Lanaspa MA. Causal or Noncausal relationship of uric acid with diabetes: table 1. Diabetes 2015;64:2720-2. 
14 Davey Smith G, Hemani G. Mendelian randomization: genetic anchors for causal inference in epidemiological studies. Hum Mol Genet 2014;23:R89-98.

15 Pfister R, Barnes D, Luben R, et al. No evidence for a causal link between uric acid and type 2 diabetes: a Mendelian randomisation approach. Diabetologia 2011;54:2561-9.

16 Sluijs I, Holmes MV, van der Schouw YT, et al. A Mendelian randomization study of circulating uric acid and type 2 diabetes. Diabetes 2015;64:3028-36.

17 Keenan T, Zhao W, Rasheed A, et al. Causal Assessment of Serum Urate Levels in Cardiometabolic Diseases Through a Mendelian Randomization Study. J Am Coll Cardiol 2016;67:407-16.

18 Wang F, Zhu J, Yao P, et al. Cohort profile: the Dongfeng-Tongji cohort study of retired workers. Int J Epidemiol 2013;42:731-40.

19 American Diabetes Association. Diagnosis and classification of diabetes mellitus. Diabetes Care 2012;35:S64-71.

20 Zhu Y, Pandya BJ, Choi HK. Prevalence of gout and hyperuricemia in the US general population: the National health and nutrition examination survey 2007-2008. Arthritis \& Rheumatism 2011;63:3136-41.

21 Levey AS, Stevens LA, Schmid CH, et al. A new equation to estimate glomerular filtration rate. Ann Intern Med 2009;150:604-12.

22 Köttgen A, Albrecht E, Teumer A, et al. Genome-Wide association analyses identify 18 new loci associated with serum urate concentrations. Nat Genet 2013;45:145-54.

23 Kamatani Y, Matsuda K, Okada Y, et al. Genome-Wide association study of hematological and biochemical traits in a Japanese population. Nat Genet 2010;42:210-5.

24 Yang B, Mo Z, Wu C, et al. A genome-wide association study identifies common variants influencing serum uric acid concentrations in a Chinese population. BMC Med Genomics 2014;7:10.

25 Okada Y, Sim X, Go MJ, et al. Meta-Analysis identifies multiple loci associated with kidney function-related traits in East Asian populations. Nat Genet 2012;44:904-9.

26 Kolz M, Johnson T, Sanna S, et al. Meta-Analysis of 28,141 individuals identifies common variants within five new loci that influence uric acid concentrations. PLoS Genet 2009;5:e1000504.

$27 \mathrm{He} \mathrm{M}, \mathrm{Wu} \mathrm{C}, \mathrm{Xu} \mathrm{J}$, et al. A genome wide association study of genetic loci that influence tumour biomarkers cancer antigen 19-9, carcinoembryonic antigen and $\alpha$ fetoprotein and their associations with cancer risk. Gut 2014;63:143-51.

$28 \mathrm{He} \mathrm{M}, \mathrm{Xu} \mathrm{M}$, Zhang B, et al. Meta-Analysis of genome-wide association studies of adult height in East Asians identifies 17 novel loci. Hum Mol Genet 2015;24:1791-800.

29 Li S, Zhao JH, Luan J, et al. Genetic predisposition to obesity leads to increased risk of type 2 diabetes. Diabetologia 2011;54:776-82.

30 Freathy RM, Timpson NJ, Lawlor DA, et al. Common variation in the FTO gene alters diabetes-related metabolic traits to the extent expected given its effect on BMI. Diabetes 2008;57:1419-26.

31 Bowden J, Davey Smith G, Burgess S. Mendelian randomization with invalid instruments: effect estimation and bias detection through Egger regression. Int J Epidemiol 2015;44:512-25.

32 Kodama S, Saito K, Yachi Y, et al. Association between serum uric acid and development of type 2 diabetes. Diabetes Care 2009;32:1737-42.

33 Lv Q, Meng X-F, He F-F, et al. High serum uric acid and increased risk of type 2 diabetes: a systemic review and meta-analysis of prospective cohort studies. PLoS One 2013;8:e56864.

34 Jia Z, Zhang X, Kang S, et al. Serum uric acid levels and incidence of impaired fasting glucose and type 2 diabetes mellitus: a meta-analysis of cohort studies. Diabetes Res Clin Pract 2013;101:88-96.
35 Bellou V, Belbasis L, Tzoulaki I, et al. Risk factors for type 2 diabetes mellitus: an exposure-wide umbrella review of meta-analyses. PLoS One 2018;13:e0194127.

36 DeFronzo RA, Ferrannini E. Insulin resistance: a multifaceted syndrome responsible for NIDDM, obesity, hypertension, dyslipidemia, and atherosclerotic cardiovascular disease. Diabetes Care 1991;14:173-94.

37 Furukawa S, Fujita T, Shimabukuro M, et al. Increased oxidative stress in obesity and its impact on metabolic syndrome. J. Clin. Invest. 2004:114:1752-61.

38 Sautin YY, Nakagawa T, Zharikov S, et al. Adverse effects of the classic antioxidant uric acid in adipocytes: NADPH oxidasemediated oxidative/nitrosative stress. Am J Physiol Cell Physiol 2007;293:C584-96.

39 Zhou $Y$, Fang $L$, Jiang $L$, et al. Uric acid induces renal inflammation via activating tubular NF- $\mathrm{KB}$ signaling pathway. PLoS One 2012; 7:e39738.

40 Duncan BB, Schmidt MI, Pankow JS, et al. Low-Grade systemic inflammation and the development of type 2 diabetes: the Atherosclerosis risk in Communities study. Diabetes 2003;52:1799-805.

41 Kaneto $\mathrm{H}$, Katakami N, Kawamori D, et al. Involvement of oxidative stress in the pathogenesis of diabetes. Antioxid Redox Signal 2007;9:355-66.

42 Tassone EJ, Cimellaro A, Perticone M, et al. Uric acid impairs insulin signaling by promoting ENPP1 binding to insulin receptor in human umbilical vein endothelial cells. Front Endocrinol 2018;9:98.

43 Nakagawa T, Hu H, Zharikov S, et al. A causal role for uric acid in fructose-induced metabolic syndrome. Am J Physiol Renal Physiol 2006;290:F625-31.

44 Ogino K, Kato M, Furuse Y, et al. Uric acid-lowering treatment with benzbromarone in patients with heart failure: a double-blind placebo-controlled crossover preliminary study. Circ Heart Fail 2010;3:73-81.

45 Niu S-W, Chang K-T, Ta A, et al. Decreased incidence of diabetes in patients with gout using benzbromarone. Rheumatology 2018:57:1574-82.

46 Waring WS, McKnight JA, Webb DJ, et al. Lowering serum urate does not improve endothelial function in patients with type 2 diabetes. Diabetologia 2007;50:2572-9.

47 Li X, Meng X, Gao X, et al. Elevated serum xanthine oxidase activity is associated with the development of type 2 diabetes: a prospective cohort study. Diabetes Care 2018;41:dc171434-890.

48 Oikonen M, Wendelin-Saarenhovi M, Lyytikäinen L-P, et al. Associations between serum uric acid and markers of subclinical atherosclerosis in young adults. the cardiovascular risk in young Finns study. Atherosclerosis 2012;223:497-503.

49 Palmer TM, Nordestgaard BG, Benn M, et al. Association of plasma uric acid with ischaemic heart disease and blood pressure: Mendelian randomisation analysis of two large cohorts. BMJ 2013;347:f4262.

50 Hall MA, Verma A, Brown-Gentry KD, et al. Detection of pleiotropy through a Phenome-wide association study (PheWAS) of epidemiologic data as part of the environmental architecture for genes linked to environment (Eagle) study. PLoS Genet 2014;10:e1004678.

51 Augustin R, Carayannopoulos MO, Dowd LO, et al. Identification and characterization of human glucose transporter-like protein- 9 (GLUT9): alternative splicing alters trafficking. Mol Membr Biol 2004;279:16229-36.

52 Manolescu AR, Augustin R, Moley K, et al. A highly conserved hydrophobic motif in the exofacial vestibule of fructose transporting SLC2A proteins acts as a critical determinant of their substrate selectivity. Mol Membr Biol 2007;24:455-63. 